\title{
Detecting Planar and Curved Symmetries of 3D Shapes from a Range Image
}

\author{
Yoshinobu Sato* and Shinichi Tamura \\ Division of Functional Diagnostic Imaging, Biomedical Research Center, Osaka University Medical School, Suita, Osaka, 565, Japan
}

Received February 22, 1994; accepted April 12, 1995

This paper addresses the problem of detecting mirror symmetries of 3D shapes. Unlike previous methods, which involve overall 3D shape data, our method uses a range image obtained from one viewpoint. Also, the proposed method can be applied to detection of local as well as global symmetries. The method consists of two stages. First, initial estimates of symmetric plane candidates are obtained using the Hough transform. As local fragments aggregated by the Hough transform, we use the local symmetry points determined by point pairs between occluding contour points and surface points. Next, the iteratively reweighted least-squares method is applied in order to perform nonmaximum suppression and refinement of the parameter values. The method is further extended so as to extract curved as well as planar symmetries. Two kinds of extensions are proposed-fitting a quadratic function instead of fitting a plane, and extracting smoothed local surface symmetries, which represent a 3D extension of smoothed local symmetries. Experimental results involving synthesized and real range images are presented. $\odot 1996$ Academic Press, Inc.

\section{INTRODUCTION}

Many objects have symmetries-both natural objects such as human faces, and man-made ones such as cars. Furthermore, even if they do not possess global symmetry, most objects have local symmetries. As a means of describing $2 \mathrm{D}$ shapes in a unique and compact manner, symmetries provide the natural axes of such shapes $[1,2]$. Also, in describing 3D shapes, shape description algorithms should recognize symmetry and encode it in the resultant descriptions. In this paper, we address the problem of detecting both global and local symmetries of 3D shapes.

There have been two main approaches to $2 \mathrm{D}$ symmetry detection, one based on moment analysis [3] and the other based on pointwise local symmetry analysis $[1,2,4]$. Al-

*E-mail: yoshi@image.med.osaka-u.ac.jp. though the former used a well-formulated theory [5, 6], a portion to be analyzed needs to be segmented and to include the overall 2D shape. Moment analysis cannot therefore be applied to locally symmetric shapes or incomplete data such as occluded shapes. On the other hand, while the latter approach is suitable both for detecting local symmetry and handling incomplete data, pointwise local symmetry detection is often sensitive to noise.

In order to identify 3D symmetry, a method based on moment analysis has been proposed for detecting various global symmetries from octree data [7]. This method, however, suffers from the same problems as 2D moment-based methods. For example, symmetry detection is impossible using a range image from one viewpoint, such as is obtained using a typical range scanner [8]. A 3D shape description scheme based on local symmetry analysis also has been reported [9], which is a $3 \mathrm{D}$ extension of the symmetric axis transform [1]. However, this work concentrated on the theoretical aspects of the scheme and did not actually demonstrate an efficient method for recovering symmetries. Furthermore, because efficient recovery in the 2D versions of the scheme involves overall shape analysis [1], it seems difficult to extend the method so as to handle incomplete 3D data. An efficient method for detecting rotational symmetries of 3D shapes has been proposed [10], which is applicable for unsegmented and incomplete data; however, this method depends on the special characteristics that solids of revolution possess.

In this paper, we describe a mirror symmetry detection method which detects both global and local symmetries of $3 \mathrm{D}$ shapes using a range image from one viewpoint. Our approach is based on a $3 \mathrm{D}$ extension of a method for detecting 2D local symmetries [2,4]. Detecting mirror symmetry of a 3D shape can be viewed as a problem of finding three parameters which determine a symmetry plane. The method for detecting 2D local symmetries [2, 4] suggests the following basic strategy for finding 3D symmetric 
planes; find symmetrical point pairs by testing all possible point pairs for the mirror symmetry constraints, and estimate the symmetric planes from symmetrical points determined by the symmetrical point pairs. However, such a straightforward extension would give rise to problems on computational cost, the reliability of symmetry detection, and the accuracy of symmetry localization. The following two improvements are therefore adopted to make the above basic strategy practical. First, we reduce the number of point pairs to be tested by considering only point pairs between occluding contour points and surface points. From a practical point of view, occluding contours have several advantageous characteristics. Because occluding contours are boundaries of visible surface, the symmetry conditions can be efficiently checked over as wide ranges as possible. Also, detected local symmetries can be easily aggregated along occluding contours into larger entities, like the recovery of 2D smoothed local symmetries. Second, we accurately estimate the parameters of local symmetric planes by combining the Hough transform and the interatively reweighted least-squares (IRLS) method [11]. The IRLS method is highly effective for both the nonmaximum suppression of peaks detected in the Hough space and refinement of the parameter values. Most importantly, the proposed method can be extended so as to extract curved symmetries on surfaces as well as planar symmetries. Shape deformation such as bending can be appropriately described by curved symmetries. We show that curved symmetries are stably extracted by any of the quadratic function and piecewise local symmetric plane fittings.

The organization of the paper is as follows: In Section 2 , we describe the conditions for checking whether point pairs between occluding contour points and surface points exhibit symmetry, and formulate an accurate and stable method for detecting planer symmetries which combines the Hough transform and a subsequent refinement process using the IRLS method. In Section 3, the method is extended so as to extract curved symmetries. Two kinds of extensions are described-fitting a quadratic function instead of a plane, and extracting smoothed local surface symmetries, which represent a 3D extension of smoothed local symmetries [2]. In Section 4, we show experimental results of planar and curved symmetry detection using both synthesized and real range images. In Section 5, we summarize the work and discuss related problems.

\section{DETECTING PLANAR SYMMETRIES}

The planar symmetry detection method consists of two stages; detection of pointwise symmetries and their aggregation. First, we describe the conditions used for detecting pointwise local symmetries from a range image and occlud-

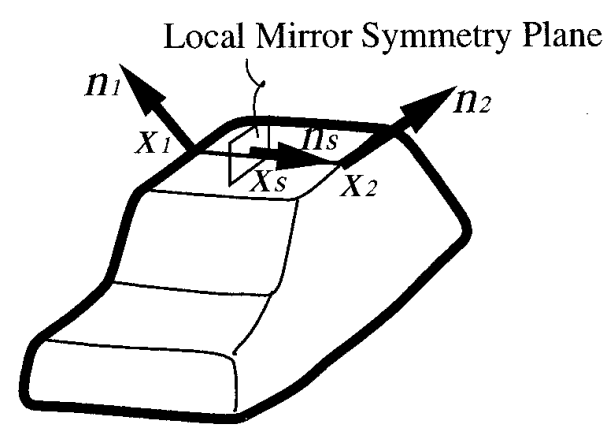

FIG. 1. Local mirror symmetry on surface.

ing contours. For each detected symmetry, the deviation from the symmetry is computed and used at the aggregation stage. We then describe the procedure for aggregating the detected pointwise symmetries into symmetric planes. Since pointwise symmetries are sensitive to noise, and there can also be multiple symmetric planes in a range image, we develop an accurate and stable method for dealing with these problems.

\subsection{Local Symmetry Conditions}

Let $\mathbf{x}_{1}$ and $\mathbf{x}_{2}$ be $3 \mathrm{D}$ positions, and $\mathbf{n}_{1}$ and $\mathbf{n}_{2}$ be surface normals at $\mathbf{x}_{1}$ and $\mathbf{x}_{2}$, respectively (Fig. 1). The mirror symmetry conditions between these two points are given by

$$
\left(\mathbf{n}_{1}+\mathbf{n}_{2}\right) \cdot\left(\mathbf{x}_{1}-\mathbf{x}_{2}\right)=0
$$

and

$$
\left(\mathbf{n}_{1}-\mathbf{n}_{2}\right)=\lambda\left(\mathbf{x}_{1}-\mathbf{x}_{2}\right),
$$

where $\lambda$ is a scalar coefficient. Symmetry point $\mathbf{x}_{s}$ and normal $\mathbf{n}_{s}$ of the local symmetry plane determined by these two points are given by

$$
\mathbf{x}_{s}=\frac{\mathbf{x}_{1}+\mathbf{x}_{2}}{2}
$$

and

$$
\mathbf{n}_{s}=\frac{\mathbf{x}_{1}-\mathbf{x}_{2}}{\left|\mathbf{x}_{1}-\mathbf{x}_{2}\right|}
$$

The symmetry conditions are checked between an occluding contour point and a surface point. Because accurate estimations of 3D positions and normals are difficult to obtain on occluding contours using surface data acquired from a range scanner, we use $2 \mathrm{D}$ occluding contours ex- 


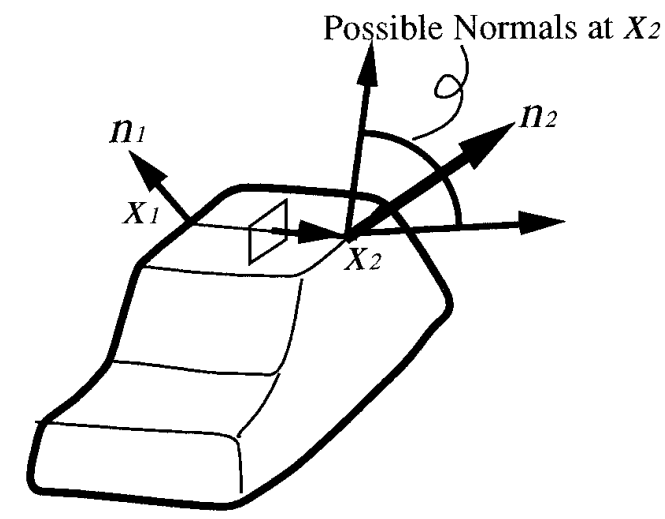

FIG. 2. Normal interpolation at a point with sudden orientation change.

tracted from a gray-level image. While the surface normal is uniquely determined at a point on the $2 \mathrm{D}$ occluding contour because the 2D occluding contour orthogonally intersects with the line of sight, the $3 \mathrm{D}$ position still has one degree of freedom along the line of sight. However, we can determine the one degree of freedom in the position by using one of the symmetry constraints (Eq. (1)), and the symmetry condition can be checked by the other constraint (Eq. (2)). When there is a surface point straddling a sudden normal change, as shown in Fig. 2, surface normals are interpolated. We assume that such a surface point can have any normal with the interpolated directions.

When two points satisfy the symmetry conditions, we check further into the symmetry of the profile shape between these two points. The profile shape is extracted by cutting a range image with the plane determined by these two points and the origin of the viewer-centered coordinate. We assume that a range scanner provides the 3D position along a line of sight corresponding to each pixel. The origin of the viewer-centered coordinate is defined as the intersection point of all the lines of sight, that is, the lens center. This condition holds for typical range scanners [8]. Let $\mathbf{u}_{1}$ and $\mathbf{u}_{2}$ be 2D image coordinates of two points satisfying the symmetry conditions, $\mathbf{X}(\mathbf{u})$ be the $3 \mathrm{D}$ position stored in pixel $\mathbf{u}$, and $\mathbf{P}(\mathbf{x})$ be the image projection of the 3D position $\mathbf{x}$. We then define the deviation from symmetry of the profile shape as

$$
\begin{aligned}
\delta= & \int_{0}^{1} \mid \mathbf{X}\left(t \mathbf{u}_{1}+(1-t) \mathbf{u}_{2}\right)+s \mathbf{n}_{s} \\
& -\left.X\left(\mathbf{P}\left(\mathbf{X}\left(t \mathbf{u}_{1}+(1-t) \mathbf{u}_{2}\right)+s \mathbf{n}_{s}\right)\right)\right|^{2} d t,
\end{aligned}
$$

where $s=2\left(\mathbf{x}_{s}-\mathbf{X}\left(t \mathbf{u}_{1}+(1-t) \mathbf{u}_{2}\right)\right) \cdot \mathbf{n}_{s}$. The intuitive explanation of Eq. (5) is shown in Fig. 3a and can be summarized as follows. Find the ideal symmetrical point $\mathbf{X}\left(t \mathbf{u}_{1}+(1-t) \mathbf{u}_{2}\right)$ (point B in Fig. 3a) paired with point
$\mathbf{X}\left(t \mathbf{u}_{1}+(1-t) \mathbf{u}_{2}\right)$ (point A in Fig. 3a) on the profile shape with respect to the detected symmetrical plane, and compute the integral of the squared distance between the ideal symmetrical point and intersection point $\mathbf{X}\left(\mathbf{P}\left(\mathbf{X}\left(t \mathbf{u}_{1}+(1-t) \mathbf{u}_{2}\right)+s \mathbf{n}_{s}\right)\right)$ of the range image surface along the line of sight corresponding to the ideal symmetrical points. If the discrete approximation of the integral is smaller than a threshold value, the profile shape is judged as symmetrical. However, if there is occlusion within the profile, this measure includes some error (Fig. 3b). In this paper, therefore, we consider the detection of only symmetries whose profile shapes do not contain occluded part.

\subsection{Accurate Estimation of Symmetric Plane Parameters}

The symmetric plane including symmetric point $\mathbf{x}_{s}$ and having normal $\mathbf{n}_{s}$ is represented as

$$
n_{x} x+n_{y} y+n_{z} z=\rho
$$

where $\mathbf{n}_{s}=\left(n_{x}, n_{y}, n_{z}\right)\left(\left|\mathbf{n}_{s}\right|=1\right)$ and $\rho=n_{s} \cdot \mathbf{x}_{s}$. Based on the concept of the Hough transform, all the local symmetry points determined by point pairs satisfying the conditions shown in Eqs. (1) and (2) are voted into the parameter

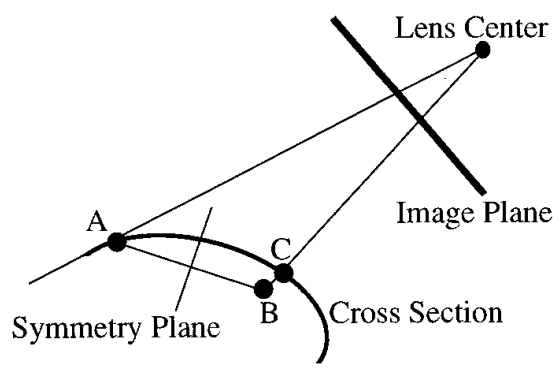

(a)

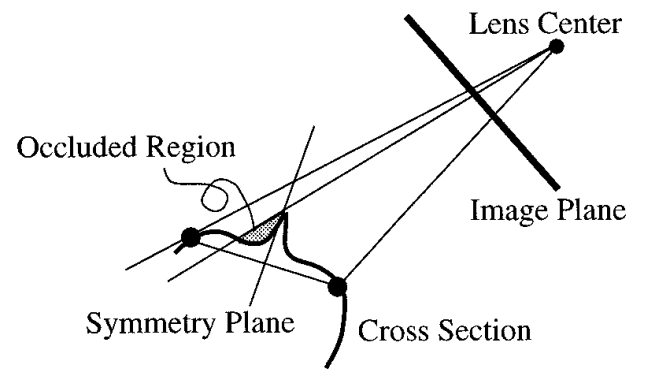

(b)

FIG. 3. Deviation from symmetry of cross-sectional shape. (a) Ideal symmetrical point and deviation from it. (b) Occluded situation. 
space. Weighted voting is performed based on the measure of the deviation from symmetry shown in Equation (5). The weight value of each vote is computed by

$$
G\left(\delta_{i} ; \sigma_{\delta}\right)
$$

where $G(x ; \sigma)=\exp \left(-x^{2} /\left(2 \sigma^{2}\right)\right), \delta_{i}$ is the deviation from symmetry computed by Eq. (5). The estimated parameters of the symmetric planes are obtained by detecting peaks in the parameter space. The parameter space of $n_{s}$ can be represented by a geodesic dome [12]. The parameter space of $\rho$ is assigned to each cell of the geodesic dome representation.

There is, however, one major drawback with the Hough method, which, is the bin size problem-i.e., deciding how finely the parameter space should be tessellated. If the tessellation is too coarse the estimation will not be accurate, but if it is too fine multiple peaks will occur around the true peak. To overcome this problem, we apply the iteratively reweighted least squares (IRLS) method [11] as a subsequent refinement process. The IRLS method iterates least square fitting with adaptive selection of the data originating from the model. By using the IRLS method the estimated parameters can be refined, while the multiple peaks which have come from the same symmetrical plane converge to almost the same parameter values.

For each detected peak in the Hough space, we apply the following refinement process. In order to formulate the problem as a regression problem, we let the symmetric plane be represented by

$$
z=\alpha_{10} x+a_{01} y+a_{00}
$$

where the $z$-axis is set so as to be in alignment with the normal of the symmetric plane corresponding to the detected peak. All the local symmetry points detected using the method described in Section 2.1 are transformed into this local coordinate system from the viewer-centered coordinate system. The symmetric plane corresponding to the detected peak is used as the initial estimates of the IRLS method. At the $m$ th iteration, symmetric plane parameter values $a_{10}^{(m)}, a_{01}^{(m)}$, and $a_{00}^{(m)}$ are reestimated by minimizing

$$
\begin{gathered}
\sum_{i} W_{i}^{(m)}\left\{\alpha\left(Z_{i}-a_{10}^{(m)} X_{i}-a_{01}^{(m)} Y_{i}-a_{00}^{(m)}\right)^{2}\right. \\
\left.+\left(P_{i}-a_{10}^{(m)}\right)^{2}+\left(Q_{i}-a_{01}^{(m)}\right)^{2}\right\}
\end{gathered}
$$

where $\left(X_{i}, Y_{i}, Z_{i}\right)$ is the $3 \mathrm{D}$ position of the symmetric point of each local symmetric plane, and $\left(P_{i}, Q_{i}, 1\right)$ represents its normal orientation. $\alpha$ is the weight parameter for the 3D positions against the normals. $W_{i}^{(m)}$ is given by

$$
W_{i}^{(m)}=G\left(\delta_{i} ; \sigma_{\delta}\right) \times G\left(\varepsilon_{i}^{(m)} ; \sigma_{\varepsilon}\right),
$$

where $G(x ; \sigma)=\exp \left(-x^{2} /\left(2 \sigma /^{2}\right)\right), \delta_{i}$ is the deviation from symmetry computed by Eq. (5), and $\varepsilon_{i}^{(m)}$ is computed by

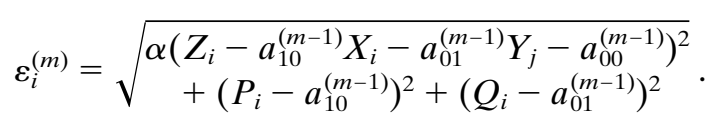

The estimates at the $(m-1)$ th iteration are used to select inliers, that is, the data originating from the model. By weighting the squared error with $G\left(\varepsilon_{i}^{(m)} ; \sigma_{\varepsilon}\right)$, only the local symmetry points which have come from the symmetry plane being estimated can contribute to the symmetry plane fitting. In the original formulation of the IRLS method, $\sigma_{\varepsilon}$ is modified at each iteration by some ad hoc procedure. In our current implementation, however, $\sigma_{\varepsilon}$ is fixed and determined according to the distribution of $3 \mathrm{D}$ points in an input range image. Another standard deviation, $\sigma_{\delta}$, is determined according to the threshold values for the measurement of the deviation from symmetry, which is also determined based on the distribution of $3 \mathrm{D}$ points in an input range image.

The strength measure of symmetry is given by

$$
\sum_{i} G\left(\delta_{i} ; \sigma_{\delta}\right) \times G\left(\varepsilon_{i} ; \sigma_{\varepsilon}\right)
$$

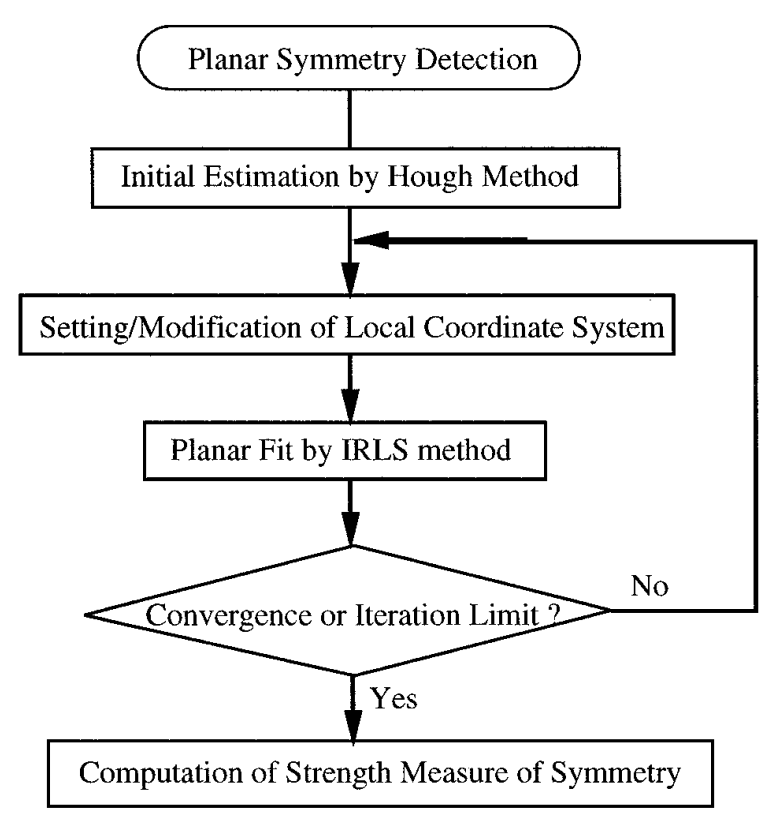

FIG. 4. Block diagram of planar symmetry detection. 
where $\varepsilon_{i}$ represents the values of Eq. (11) at the final iteration. This measure corresponds to the amount of supports for the estimated symmetric plane. Although the strength measure of symmetry is not a normalized value, the measure can be used to compare the relative strengths among detected symmetries.

Because the residual is measured along the $z$-axis direction at each step of the IRLS fitting, the resultant symmetric plane parameters and strength measure of symmetry are corrdinate-system-dependent. The coordinate system for the IRLS fitting is determined based on the symmetric plane parameter values corresponding to the peak in the Hough space. As described earlier, there can be the multiple peaks that originate from the same symmetrical plane. Thus, different coordinate systems may be chosen for fitting the same symmetric plane, and then different values may be estimated for the same symmetry. In order to avoid this situation, the corrdinate system of the planer fit is iteratively modified. At each iteration, the coordinate system is updated so that the $z$-axis is in alignment with the normal of the plane estimated at the previous planar fitting. This coordinate system modification is iterated to reach convergence or the iteration limit (10 times in our implementation). We confirmed experimentally that this iteration usually reaches convergence within 10 times. Figure 4 shows a block diagram summarizing the method.

\section{EXTRACTING CURVED SYMMETRIES}

The method described in Section 2 can be extended so as to detect curved as well as planar symmetries. We consider two possible approaches to such an extensionfitting a quadratic function instead of a plane, and the extraction of smoothed local surface symmetries, which represent a 3D extension of smoothed local symmetries [2]. The former is appropriate for recognizing globally curved symmetries; the latter, although a little computationally expensive, makes it explicit where on the surface local symmetries exist.
3.1. Estimating a Symmetric Surface Represented as a Quadratic Function

A quadratic function can be represented as

$$
\begin{aligned}
z= & \sum_{k+\ell \leq 2} a_{k \ell} x^{k} y^{\ell}=a_{20} x^{2}+a_{11} x y \\
& +a_{02} y^{2}+a_{10} x+a_{01} y+a_{00} .
\end{aligned}
$$

Its partial derivatives are given by

$$
\frac{\partial z}{\partial x}=\sum_{k \neq 0} k a_{k \ell} x^{k-1} y^{\ell}=2 a_{20} x+a_{11} y+a_{10},
$$

and

$$
\frac{\partial z}{\partial y}=\sum_{\ell \neq 0} \ell a_{k \ell} x^{k} y^{\ell-1}=a_{11} x+2 a_{02} y+a_{01} .
$$

Equation (9) is modified so as to fit the quadratic function. At the $m$ th iteration, coefficients $a_{k \ell}^{(m)}$ of the quadratic function are estimated by minimizing

$$
\begin{aligned}
& \sum_{i} W_{i}^{(m)}\left\{\alpha\left(Z_{i}-\sum_{k+\ell \leq 2} a_{k \ell}^{(m)} X_{i}^{k} Y_{i}^{\ell}\right)^{2}\right. \\
& +\left(P_{i}-\sum_{k \neq 0} k a_{k \ell}^{(m)} X_{i}^{k-1} Y_{i}^{\ell}\right)^{2} \\
& +\left(Q_{i}-\sum_{\ell \neq 0} \ell a_{k \ell}^{(m)} X_{i}^{k} Y_{i}^{\ell-1}\right)^{2} \\
& \left.+\beta \sum_{k+\ell=2}\left(a_{k \ell}^{(m)}\right)^{2}\right\}
\end{aligned}
$$

where $\sum_{k+\ell=2}\left(a_{k \ell}^{(m)}\right)^{2}$ is introduced in order to stabilize the second order coefficients. $W_{i}^{(m)}$ is given by

$$
W_{i}^{(m)}=G\left(\delta_{i} ; \sigma_{\delta}\right) \times G\left(\varepsilon_{i}^{(m)} ; \sigma_{\varepsilon}\right),
$$

where

$$
\varepsilon_{i}^{(m)}=\sqrt{\alpha\left(Z_{i}-\sum_{k+\ell \leq 2} a_{k \ell}^{(m-1)} X_{i}^{k} Y_{i}^{\ell}\right)^{2}+\left(P_{i}-\sum_{k \neq 0} k a_{k \ell}^{(m-1)} X_{i}^{k-1} Y_{i}^{\ell}\right)^{2}+\left(Q_{i}-\sum_{\ell \neq 0} \ell a_{k \ell}^{(m-1)} X_{i}^{k} Y_{i}^{\ell-1}\right)^{2}} .
$$

As the initial values of $a_{10}, a_{01}$, and $a_{00}$, symmetric plane parameters estimated by minimizing Eq. (9) are used. The initial values of $a_{20}, a_{11}$, and $a_{02}$ are zero. Similarly, higherorder polynomials can be fitted using the second-order coefficients as the initial values.

The final step is the judgement for selecting the planar or the quadratic fit. One method is simply evaluating $a_{20}^{2}+a_{11}^{2}+a_{20}^{2}+a_{02}^{2} \approx 0$. The other method is to examine the strength measure of symmetry shown in Eq. (12). If this measure is significantly larger in the quadratic than in the planar fit, the quadratic fit should be selected. Otherwise, the planar fit should be used. In the former method, the threshold value used for the judgement should be dependent on the area and shape of the distribution of symmetric points from which each detected symmetry surface originated. Therefore, appropriate normalization is needed 


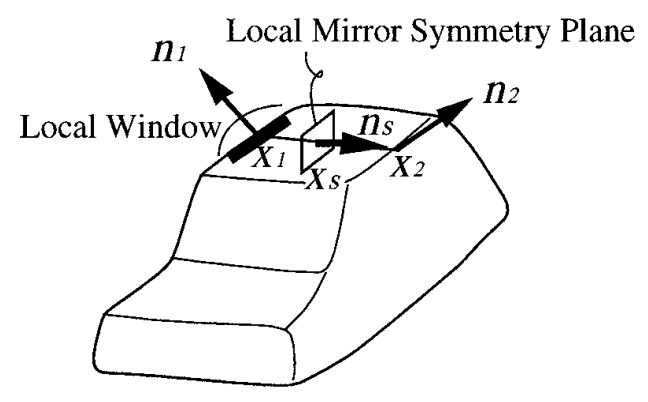

FIG. 5. Piecewise extraction of local symmetric planes.

for each symmetry as well as for each image. On the other hand, such normalization is not necessary if we use the ratios of the strength measures of symmetry of the planer fit to the quadratic fit. Therefore, in this paper, we use the strength measures of symmetry for this selection.

\section{2. $\quad$ Extracting Smoothed Local Surface Symmetries}

The detection of curved symmetries can be also realized by applying the planar symmetry detection method only to the occluding contour points within a local window (with five-point width in our experiments). As shown in Fig. 5, we consider the local window at every point on the occluding contour. At each point, the Hough transform is performed based on pointwise local symmetry checking between the surface points and the occluding contour points within the local window. Accurate planar symmetry parameters can then be estimated after the refinement process for the data within the local window. The curved symmetries can be extracted by linking local planar symmetries detected at each point along the occluding contour. Currently, we do not use any sophisticated grouping procedure which incorporates the inaccuracy of the symmetry plane parameters and spurious local symmetries, such as dynamic programming or a heuristic search for a linking process, because the local symmetries detected using the subsequent refinement process are accurate and unambiguous enough for them to be linked only by checking similarities between the symmetric plane parameters at two adjacent occluding contour points. The conditions for linking two adjacent local symmetries are

$$
\begin{gathered}
\left|\mathbf{n}_{s}-\mathbf{n}_{s}^{\prime}\right|<T_{n}, \\
\left|\mathbf{X}_{s}-\mathbf{X}_{s}^{\prime}\right|<T_{p},
\end{gathered}
$$

where $\mathbf{n}_{s}$ and $\mathbf{n}_{s}^{\prime}$ are the normals of symmetry planes estimated at the adjacent occluding points, and $\mathbf{X}_{s}$ and $\mathbf{X}_{s}^{\prime}$ are the 3D positions of local symmetries at adjacent points. $T_{n}$ and $T_{p}$ are the thresholds for the linking. We call the extracted sequences of local symmetries, which can be regarded as a 3D extension of the smoothed local symmetries for 2D contours [2], "smoothed local surface symmetries."

\section{EXPERIMENTAL RESULTS}

We evaluated the methods using synthesized and real range images. The methods was implemented on a Sun Sparcstation 10 (Model 30). Normal images were computed by local plane fitting at each point of the range images. Occluding contours were extracted from an intensity image in the case of using a real range image, and from the range image itself in the case of using a synthesized range image. The extracted occluding contours were approximated by line segments and each line segment was further divided so as to have almost the same length. The midpoint of each divided line segment was considered as an occluding contour point, and the $2 \mathrm{D}$ orientation and position of the divided line segment were used to compute a 3D normal at the point. In the following experiments, the number of extracted occluding contour points was around fifty. The image size was $128 \times 128$ or $128 \times 120$ (pixels). A computation time of about two minutes required for estimating the symmetric plane and quadratic parameters, and about 10 min for extracting the smoothed local surface symmetries.

The method includes several parameters to be tuned. In the experiments, the symmetry condition of Eq. (2) was checked as follows:

$$
\frac{\mathbf{n}_{1}-\mathbf{n}_{2}}{\left|\mathbf{n}_{1}-\mathbf{n}_{2}\right|} \frac{\mathbf{x}_{1}-\mathbf{x}_{2}}{\left|\mathbf{x}_{1}-\mathbf{x}_{2}\right|}<0.05
$$

The threshold value for the deviation from symmetry, $T_{\delta}$, was $0.05 \times \sigma_{p}$, where $\sigma_{p}$ is the standard deviation of all the $3 \mathrm{D}$ positions of the range image. $\sigma_{p}$ was used to normalize the object scale. The geodesic dome of the Hough space was tessellated into 320 triangular cells to represent $\mathbf{n}_{s}$, in Eq. (6), and each triangular cell was assigned to a linear array having 32 cells to represent $\rho$. The maximum and minimum values of $\rho$ were $2 \times \sigma_{p}$ and $-2 \times \sigma$, respectively. Possible symmetry planes were represented in the coordinate system whose origin was the centroid of all the 3D positions of the range image. In IRLS fitting of the plane and quadratic function, the weight parameter $\alpha$ for the 3D position data against the normals data was $1 / \sigma_{p}^{2}$. The weight parameter $\beta$ for stabilizing the quadratic fit was $\sigma_{p}^{2}$. In the final least-squares fitting, however, $\beta$ was zero in order to compute the unbiased variance estimate appropriately. The standard deviations of the Gaussian function, $\sigma_{\delta}$ and $\sigma \varepsilon$, to determine the weight $W_{i}^{m}$, were $T_{\delta} / 3$ and 0.1 , respectively. When we extract smoothed local surface symmetries we require the threshold values of Eqs. (19) and (20). These were $T_{n}=0.15$ and, $T_{p}=0.05 \times \sigma_{p}$. 


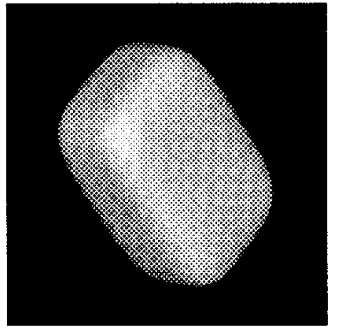

(a)

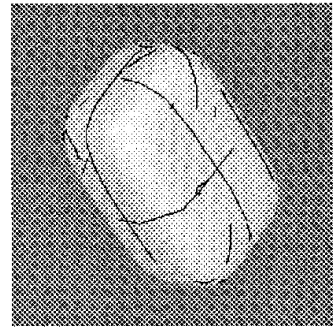

(e)
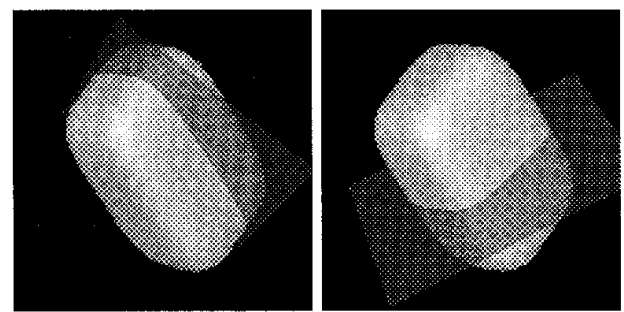

(b)
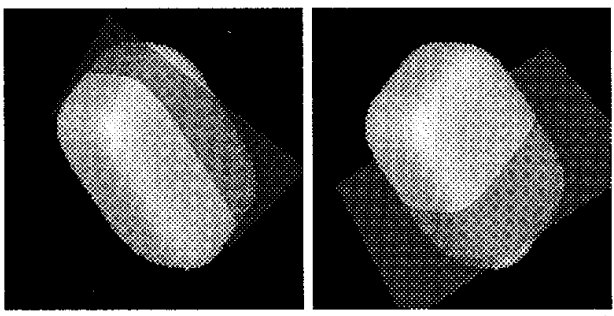
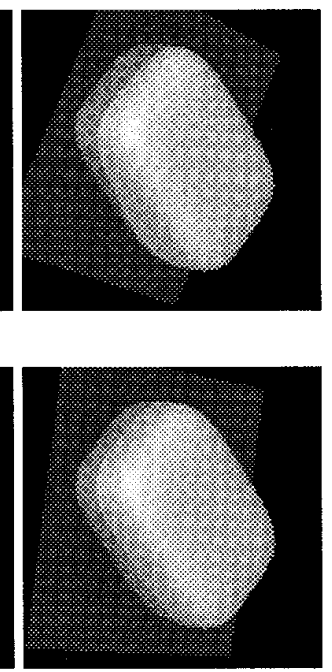

(c)
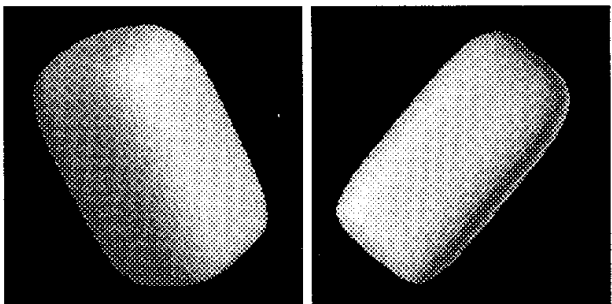

(d)
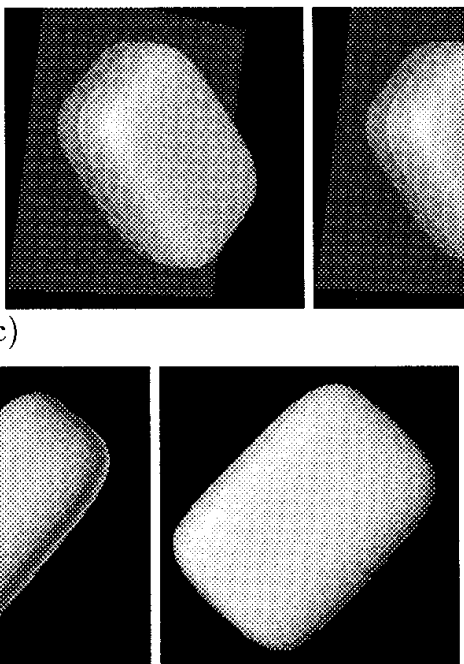

FIG. 6. Results of superquadric image. The detected symmetric planes are displayed as transparent surfaces, and superimposed with input range image surface. (a) Shaded display of input range image. (b) Initial estimates of symmetric planes by the Hough method. The results are lined up from left to right in order of height of the peaks detected by the Hough method. (c) Refined estimates of symmetric planes by the IRLS method. Each result was obtained from the initial estimate shown just above it. (d) Recovered axial volumetric model. Oblique view (left) and side views (middle and right) from the directions corresponding to the normals of two symmetric planes which determine the axis, which is set to the diagonal direction in images. (e) Smoothed local surface symmetries.

As described above, all the parameter values were normalized by the object scale using $\sigma_{p}$. In the experiments, we tried several degrees of fineness in the tessellation of the Hough space. However, the final estimated parameters were almost the same irrespective of the fineness of the tessellation.

\subsection{Superquadric Image}

In the first experiment, we used a synthesized image of a superquadric to evaluate the accuracy of the estimation of the symmetric plane parameters. The image size was $128 \times 128$ (pixels). Figure 6a shows the shaded image. This superquadric has three global mirror symmetries. Figure 6b shows the symmetric planes corresponding to the four highest peaks detected by the Hough transform. Figure 6c shows the estimated symmetric planes after the refinement process. Table 1 gives the estimated symmetric plane parameters before and after the refinement process, and the true symmetric plane parameters of the three principle planes of the superquadric. After the refinement process, more accurate estimates could be obtained. Also, the initial 
TABLE 1

Initial Estimates, Refined Estimates, and True Values of Symmetric Plane Parameters

\begin{tabular}{ccccc}
\hline $\begin{array}{c}\text { Peak } \\
\text { no. }\end{array}$ & Initial & $\begin{array}{c}\text { Refined } \\
\text { estimates }\end{array}$ & $\begin{array}{c}\text { True } \\
\text { values }\end{array}$ \\
\hline 1 & $n_{x}$ & 0.547 & 0.542 & 0.550 \\
& $n_{y}$ & -0.707 & -0.705 & -0.700 \\
& $n_{z}$ & 0.448 & 0.456 & 0.455 \\
& $\rho$ & 115.0 & 116.8 & 116.4 \\
2 & $n_{x}$ & 0.401 & 0.458 & 0.455 \\
& $n_{y}$ & 0.798 & 0.701 & -0.708 \\
& $n_{z}$ & 0.450 & 0.547 & 0.540 \\
& $\rho$ & 115.8 & 140.0 & 138.3 \\
3 & $n_{x}$ & -0.647 & -0.702 & -0.700 \\
& $n_{y}$ & 0.000 & -0.091 & -0.091 \\
& $n_{z}$ & 0.762 & 0.706 & 0.708 \\
& $\rho$ & 194.8 & 180.9 & 181.3 \\
4 & $n_{x}$ & -0.707 & -0.702 & 0.700 \\
& $n_{y}$ & -0.303 & -0.091 & -0.091 \\
& $n_{z}$ & 0.639 & 0.706 & 0.708 \\
& $\rho$ & 164.5 & 180.9 & 181.3 \\
\hline
\end{tabular}

estimates which came from the same symmetric plane (the third and the fourth peaks) could converge to the same parameter values. Table 2 shows the strength measures of symmetry for the detected three symmetries. Figure $6 \mathrm{~d}$ shows the recovery result of an axial volumetric model based on the two symmetric planes having the larger values of the strength measures of symmetry. An axial volumetric model can be recovered by the method described in [13], when the shape has two global symmetric surfaces which intersect orthogonally, and can be approximated by a generalized cone whose cross section shape is scaled differently in the two orthogonal directions on the cross section plane along an axis determined as the intersection line of the two symmetric surfaces. Figure 6e shows the smoothed local surface symmetries. Several local symmetries were extracted as well as global symmetries. Also, the locations of local symmetries on the surface could be made explicit.

\subsection{Deformed Superquadric Image}

In the second experiment, we used images of deformed superquadrics in order to evaluate the tolerance for the curvature of a symmetric surface in attempting to fit a quadratic surface from an initial fit of a plane. Figure 7

TABLE 2

Strength Measure of Symmetries

\begin{tabular}{cc}
\hline Peak no. & Strength measure of symmetry \\
\hline 1 & 1.81 \\
2 & 0.93 \\
3,4 & 2.01 \\
\hline
\end{tabular}

shows the input images of deformed superquadrics and the detected curved symmetric surfaces with different curvatures. Each superquadric shown in Fig. 7 was generated by combining an isotropic bend along a principle axis using the method described in [16]. As shown in Fig. 7f, the detection of a symmetric surface with high curvature failed. The result of quadratic fitting got trapped in other peripheral local symmetry. In this case, the symmetric plane used as an initial fit of quadratic fit had already got trapped in peripheral symmetry (Fig. 8).

In order to evaluate the tolerance for the curvature quantitatively, we plotted the relation between curvature $\kappa$ of deformed superquadrics and $a_{20}^{2}+a_{11}^{2}+a_{02}^{2}$ estimated by quadratic fitting in Fig. 9. If appropriate quadratic symmetric surfaces have been found, $a_{20}^{2}+a_{11}^{2}+a_{02}^{2}$ should be roughly proportional to $\kappa^{2}$. In Fig. 9, the relation $a_{20}^{2}+a_{11}^{2}+a_{02}^{2} \propto \kappa^{2}$ can be observed from $\kappa=0.0$ to $\kappa=0.035$. In other words, it can be considered that the estimation procedure worked well to $\kappa=0.035$.

We also plotted the relation between curvature $\kappa$ of deformed superquadrics and the ratios of the strength measures of symmetry (SMS) of the planer fit to the quadratic fit in Fig. 10. As described in the end of Section 3.1, the ratio of SMS of the planar to the quadratic fit can be used as a normalized measure for the judgement for selecting the planar or the quadratic fit. We used 0.95 as an empirically determined threshold value in the following experiments. That is, the quadratic fit was selected if the ratio of SMS of the planar to the quadratic fit was less than 0.95 .

\subsection{Handset Image}

Figure 11 shows the results of the Handset image. The image size was $128 \times 120$ (pixels). In this experiment, we used data acquired from a range scanner using lightstriping [8]. We show the detected symmetries whose strength measures of symmetry (SMS) were more than half of the highest value among all the detected symmetries. Table 3 shows the strength measures of symmetry of detected symmetries, and the results on the selection of planar or quadratic fit. Interestingly, the quadratic fit result having the second highest SMS seems to track the deformation of this handset shape accurately. In this case, when the quadratic fit was selected, the strength measure of symmetry was significantly increased as shown in Table 3. For the other symmetries, the planar fit was selected because these two measurements were almost the same. Figure 11e shows the smoothed local surface symmetries. The method could extract many of the local symmetries that this handset shape possesses.

\subsection{Deformed Handset Image}

In this experiment, the input range image was a deformed version of the axial representation obtained from 

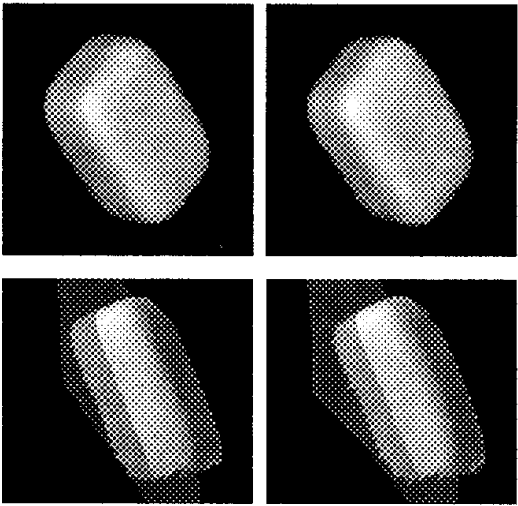

(a)

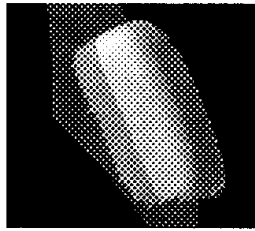

(b)
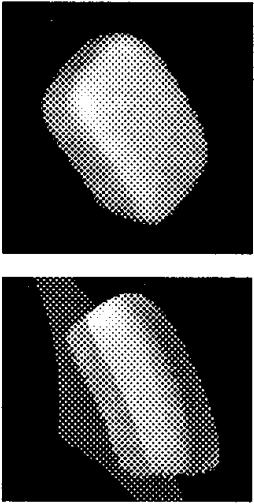

(c)
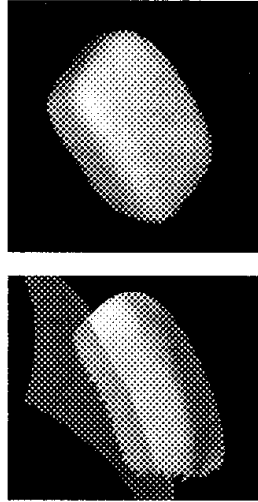

(d)
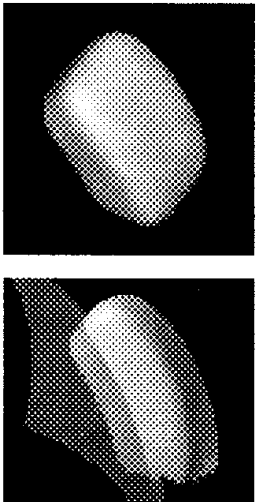

(e)
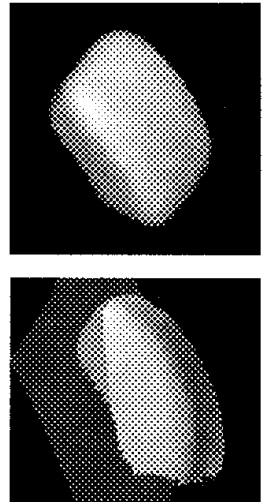

(f)

FIG. 7. Results of deformed superquadric images. Input images (top) and detected symmetric surfaces (bottom). We show the results of symmetry detection by images viewed from a different viewpoint so as to illustrate clearly the location of the detected symmetric surface. The lengths of three principle axes of the superquadric were 12,8 , and 6 , and the squareness parameter was 0.5 . (a) $\kappa=0$; (b) $\kappa=0.01$; (c) $\kappa=0.02$; (d) $\kappa=0.03$; (e) $\kappa=0.04$; (f) $\kappa=0.05$.

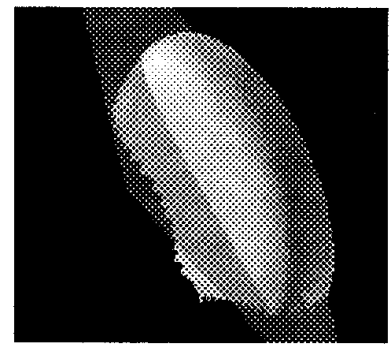

(a)

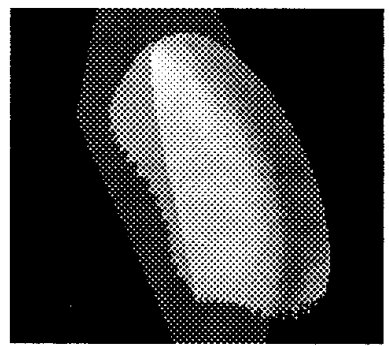

(b)

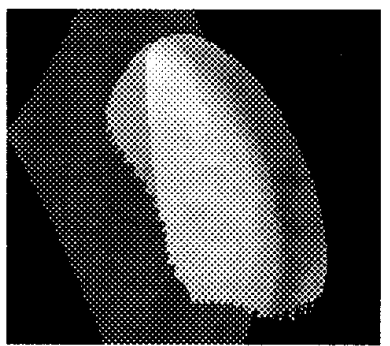

(c)

FIG. 8. Results of deformed superquadric image with $\kappa=0.05$. (a) Initial estimate of symmetric planes by the Hough method. (b) Refined estimate of symmetric planes by the IRLS method. (c) Final estimate of quadratic fitting.

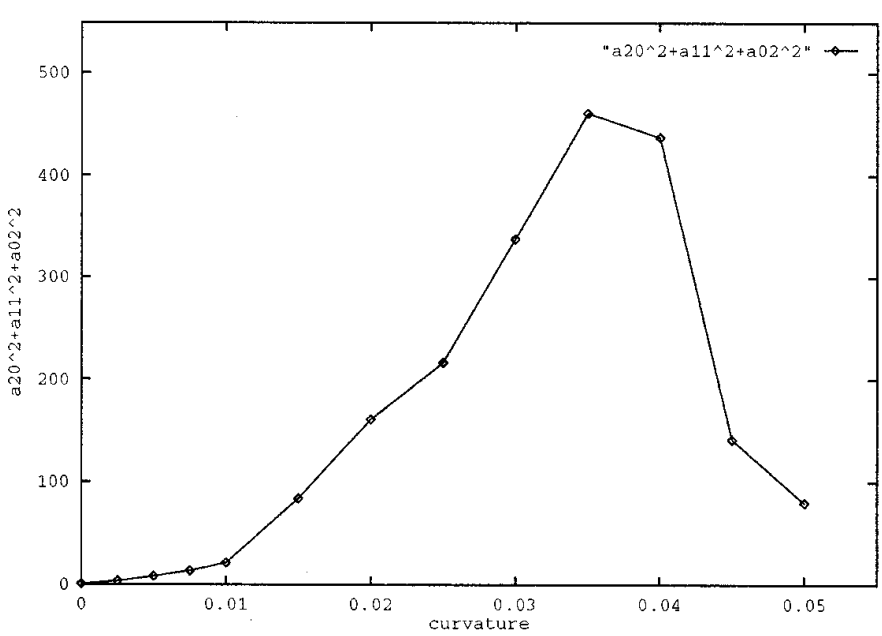

FIG. 9. Relation between curvature $\kappa$ of deformed superquadric and $a_{20}^{2}+a_{11}^{2}+a_{02}^{2}$.

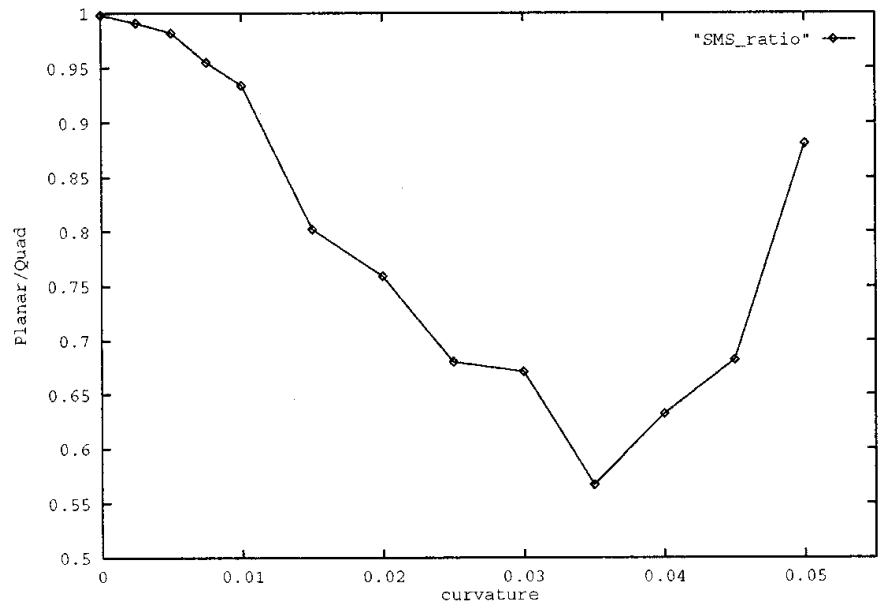

FIG. 10. Relation between curvature $\kappa$ of deformed superquadric and the ratio of the strength measure of symmetry (SMS) of planar to quadratic fit. 


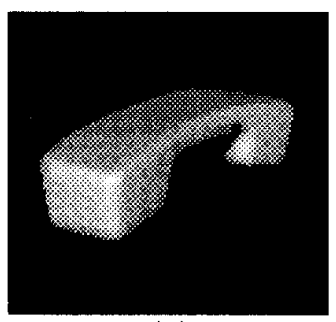

(a)

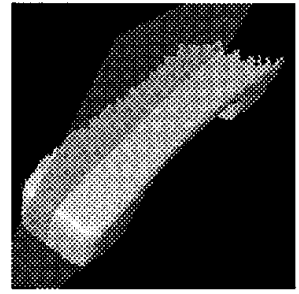

(b)

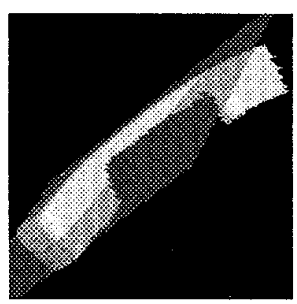

(c)

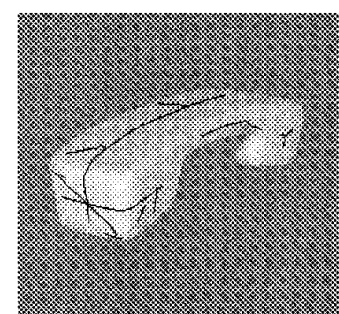

(e)

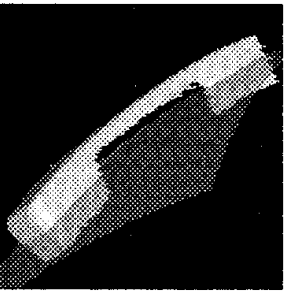

c)

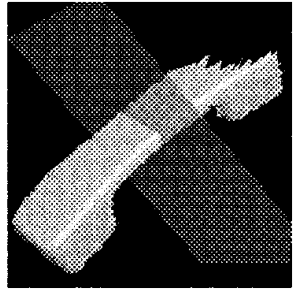

(d)

FIG. 11. Results of Handset image. We show the results of symmetry detection by an image from a different viewpoint for each symmetry so as to illustrate clearly the location of the detected symmetry. (a) Shaded display of input range image. (b) Detected planar symmetry with the highest SMS. (c) Detected planar (left) and curved (right) symmetries with the second highest SMS. (d) Detected planar symmetry with the third highest SMS. (e) Smoothed local surface symmetries.

the range image of Fig. 11a using the method described in [13]. The input image was generated by giving a bend to the extracted axis of the axial volumetric model. In Fig. 12, we show detected symmetries whose SMS was more than half of the highest SMS. The quadratic fit result with the highest SMS shown in Fig. 12b seems track the deformation of this shape. Figure $12 \mathrm{~d}$ shows the smoothed local surface symmetries.

\subsection{Face Image}

In the following two experiments, we used data acquired from a panoramic range scanner [14]. Given the panoramic data and viewpoint, an input range image was generated. The image size was $128 \times 128$ (pixels). Figure 13 shows the results of the Face sculpture image. The detected symmetry having the highest value of SMS captured the symmetry of this Face sculpture. In this case, we only show one detected symmetry (Fig. 13b) because the values of SMS of other symmetries were much smaller than the highest one (less than one-third). Figure 13c shows the smoothed local surface symmetries of the Face sculpture image. Because the upper part of this Face sculpture has a roughly rotational symmetric shape, some detected local symmetries do not agree with our intuition.

\subsection{Detergent Bottle Image}

Figure 14 shows the results with a Detergent Bottle, which has two global symmetries. We show detected sym-

TABLE 3

Results of the Selection of Planar or Quadratic Fit Using the Strength Measure of Symmetry

\begin{tabular}{|c|c|c|c|c|c|}
\hline \multirow[b]{2}{*}{ Object } & \multirow[b]{2}{*}{ Symmetry } & \multicolumn{2}{|c|}{$\begin{array}{l}\text { Strength measure } \\
\text { of symmetry }\end{array}$} & \multirow{2}{*}{$\begin{array}{c}\text { Ratio } \\
\text { (planar/quadratic) }\end{array}$} & \multirow{2}{*}{$\begin{array}{l}\text { Planar or } \\
\text { quadratic }\end{array}$} \\
\hline & & Planar & Quadratic & & \\
\hline \multirow[t]{3}{*}{ Handset } & Fig. 11b & 2.04 & 2.05 & 1.00 & Planar \\
\hline & Fig. 11c & 1.70 & 1.91 & 0.88 & Quadratic \\
\hline & Fig. 11d & 1.31 & 1.31 & 1.00 & Planar \\
\hline \multirow[t]{2}{*}{ Deformed Handset } & Fig. 12b & 1.06 & 1.21 & 0.88 & Quadratic \\
\hline & Fig. 12c & 0.82 & 0.82 & 1.00 & Planar \\
\hline Face & Fig. 13b & 2.93 & 2.99 & 0.98 & Planar \\
\hline \multirow[t]{2}{*}{ Detergent Bottle } & Fig. 14b & 6.48 & 6.65 & 0.97 & Planar \\
\hline & Fig. 14c & 4.14 & 4.15 & 1.00 & Planar \\
\hline
\end{tabular}




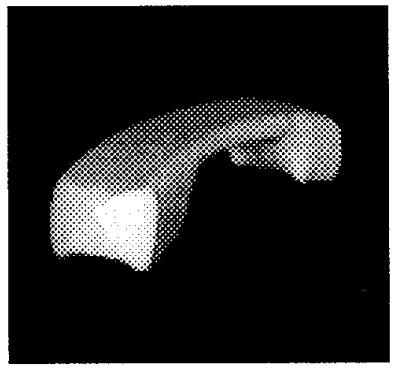

(a)

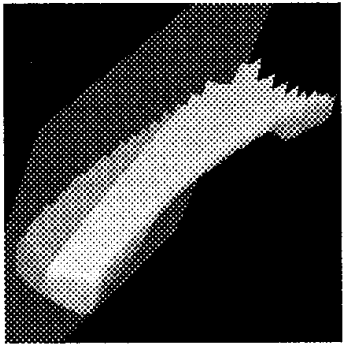

(b)

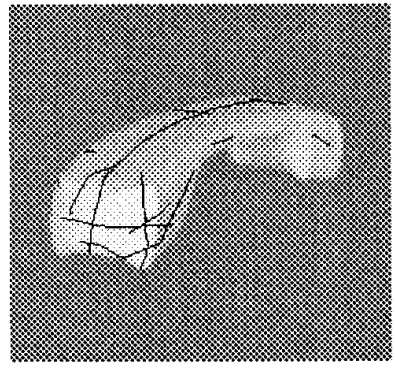

(d)

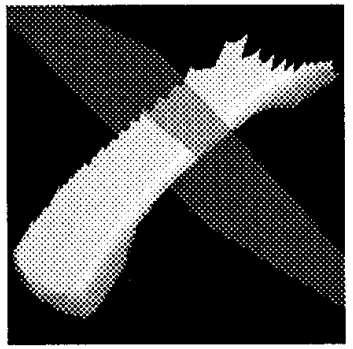

(c)

FIG. 12. Results of Deformed Handset image. We show the results of symmetry detection by an image from a different viewpoint for each symmetry so as to illustrate clearly the location of the detected symmetry. (a) Shaded display of input range image. (b) Detected planar (left) and curved (right) symmetries with the highest SMS. (c) Detected planar symmetry with the second highest SMS. (d) Smoothed local surface symmetries.

metries whose SMS was more than half of the highest SMS. The method could detect two global symmetric planes that this object possesses. Figure $14 d$ shows a recovery result of an axial volumetric model whose axis is the intersection line of the two symmetric planes.

\section{CONCLUSION}

We have described methods for detecting global and local symmetries on surfaces from a range image. The initial estimates of the symmetric plane parameters are obtained using the Hough transform based on point pairs of occluding contour and surface points. The symmetry parameters are accurately reestimated by the subsequent refinement process using the iteratively reweighted least square method. We have shown that the refinement process is useful for nonmaximum suppression as well as for accurate parameter estimation. We have also demonstrated two extensions of the method for extracting curved symmetries. The first involves the use of a quadratic instead of a planer fit. The second method for extracting curved symmetries employs smoothed local surface symmetries, which can be regarded as a $3 \mathrm{D}$ extension of smoothed local symmetries [2] for 2D contours. It has been shown that

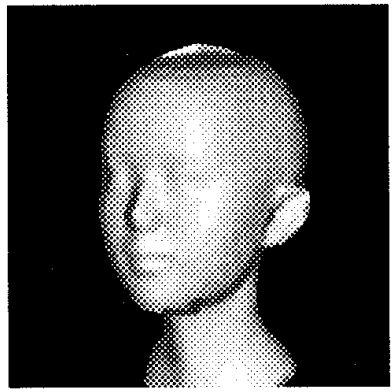

(a)

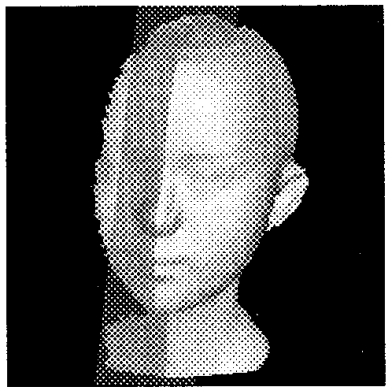

(b)

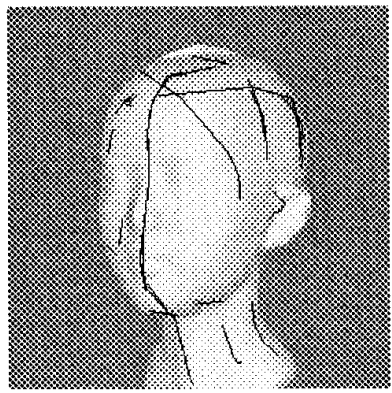

(c)

FIG. 13. Results of Face sculpture image. (a) Shaded display of input range image. (b) Detected planar symmetry with the highest SMS. We show the result of symmetry detection by an image from a different viewpoint so as to illustrate clearly the location of the detected symmetry. (c) Smoothed local surface symmetries. 


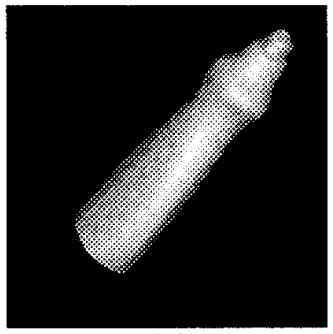

(a)
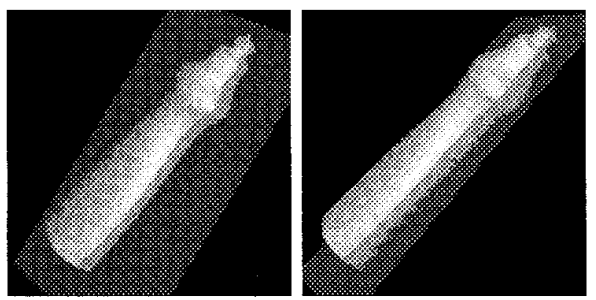

(b)

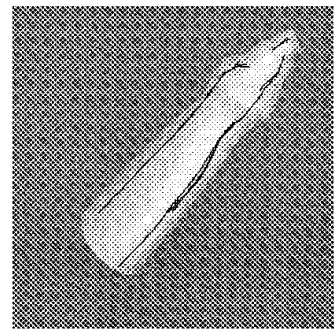

(e)
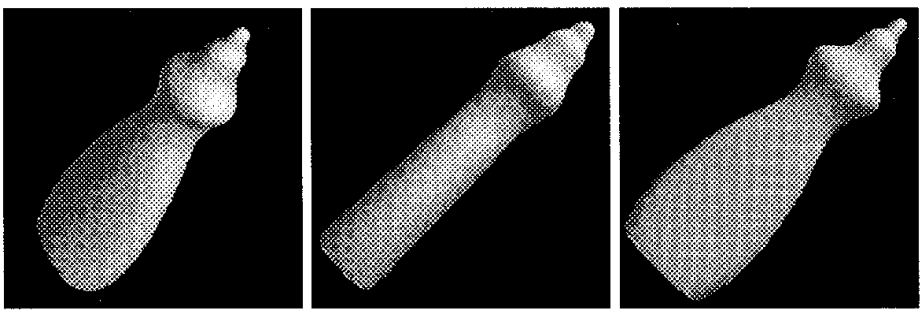

(d)
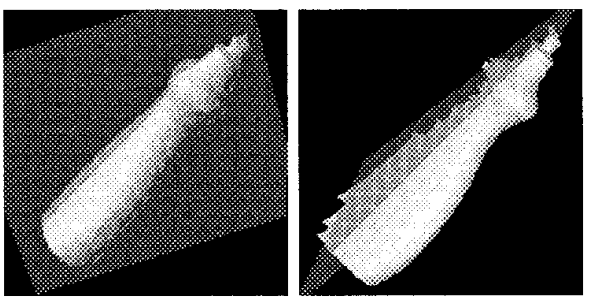

(c)

FIG. 14. Results of Detergent Bottle image. We show the results of symmetry detection by images from a different viewpoint for each symmetry (right), as well as the viewpoint from which the input was taken (left), so as to illustrate clearly the location of the detected symmetries. (a) Shaded display of input range image. (b) Detected planar symmetry with the highest SMS. (c) Detected planar symmetry with the second highest SMS. (d) Recovered axial volumetric model. Oblique view (left) and side views (middle and right) from the directions corresponding to the normals of two symmetric planes which determine the axis, which is set to the diagonal direction in images. (e) Smoothed local surface symmetries.

local symmetries can only be generated by extrema of curvature along a line of curvature [15]. Although curvature extrema having high absolute curvature values can be localized using curvature estimates from local window data, the localization is unstable for curvature extrema having low absolute values using curvature estimates. The method proposed here is suitable for extracting local symmetries generated by curvature extrema having low absolute values. While extrema having high absolute curvature can be considered as the boundaries of segmented surface patches, those having low absolute curvature can be considered as the descriptors of segmented surface patches. The experimental results on extracting the smoothed local surface symmetries have potential for use as such surface descriptors.

Although several methods have recently been proposed for recovering viewpoint-independent volumetric descriptions from images [17-20], the procedures used are highly dependent on nonlinear optimization, and therefore the recovery of descriptions is often unstable. The difficulty with these methods is caused by the simultaneous processing of shape recovery and determination of the coordinate system in which the recovered shape is described. If the coordinate system is determined beforehand, however, the shape recovery problem becomes much easier. Symmetries can be important cues for the determination of the coordinate system. We believe that symmetries extracted by the methods proposed here can be used for the stable recovery of volumetric descriptions such as generalized cone representation [13].

\section{ACKNOWLEDGMENTS}

A part of this work was conducted at NTT Human Interface Laboratories. The authors thank Dr. Jun Ohya and Dr. Kenichiro Ishii for their support while the first author was at NTT.

\section{REFERENCES}

1. H. Blum, Biological shape and visual science (part I), J. Theor. Biol. 38, 1973, 205-287. 
2. M. Brady and H. Asada, Smoothed local symmetries and their implementation, Int. J. Robotics Res. 3(3), 1984, 36-60.

3. S. A. Friedberg, Finding axes of skewed symmetry, Comput. Vision Graphics Image Process. 34, 1986, 138-155.

4. J. Ponce, On characterizing ribbons and finding skewed symmetries, Comput. Vision Graphics Image Process. 52, 1990, 328-340.

5. H. Weyl, Symmetry, Princeton Univ. Press, Princeton, NJ, 1952.

6. K. R. Symon, Mechanics, Addison-Wesley, Reading, MA, 1965.

7. P. Minovic, S. Ishikawa, and K. Kato, Symmetry identification of a 3-D object represented by octree, IEEE Trans. Pattern Anal. Mach. Intell. 15(5), 1993, 507-514.

8. K. Sato and S. Inokuchi, Range-imaging system utilizing nematic liquid crystal mask, in Proc. 1st International Conference on Computer Vision, London, 1987, pp. 657-661.

9. L. R. Nackman, Three-dimensional shape description using the symmetric axis transform-I: Theory, IEEE Trans. Pattern Anal. Mach. Intell. PAMI-7(2), 1985, 187-202.

10. N. Yokoya and D. Levine, Volumetric description of solids of revolution in a range image, in Proc. 10th International Conference on Pattern Recognition, Atlantic City, NJ, 1990, pp. 303-308.

11. P. J. Besl, J. B. Birsh, and L. T. Watson, Robust window operator, in Proc. 2nd International Conference on Computer Vision, Tampa, FL, 1988, pp. 591-600.
12. B. K. P. Horn, Robot Vision, Chap. 16, MIT Press, Cambridge, MA, 1986.

13. Y. Sato, J. Ohya, and K. Ishii, Smoothed local generalized cones: An axial representation of 3D shapes, in Proc. IEEE Conference on Computer Vision and Pattern Recognition, Champaign, IL, 1992, pp. $56-62$.

14. Y. Suenaga and Y. Watanabe, A method for the synchronized acquisition of cylindrical range and color data, in Proc. IAPR Workshop on Machine Vision Application, 1990, pp. 137-141.

15. A. Yuille and M. Leighton, 3D symmetry-curvature duality theorems, in Proc. 1st International Conference on Computer Vision, London, 1987, pp. 721-726.

16. A. H. Barr, Global and local deformation of solid primitive, Comput. Graphics 18(3), 1984, 21-30.

17. A. P. Pentland, Recognition by parts, in Proc. 1st International Conference on Computer Vision, London, 1987, pp. 612-620.

18. F. Solina and R. Bajcsy, Recovery of parametric models from range images: The case for superquadrics with global deformations, IEEE Trans. Pattern Anal. Mach. Intell. PAMI-12(2), 1990, 131-146.

19. D. Terzopoulos, A. Witkin, and M. Kass, Symmetry-seeking models for 3D object reconstruction, in Proc. 1st International Conference on Computer Vision, London, 1987, pp. 269-276.

20. D. Terzopoulos and D. Metaxas, Dynamic 3D models with local and global deformation: Deformable superquadrics, in Proc. 3rd International Conference on Computer Vision, Osaka, 1990, pp. 606-615. 